\title{
Nanoemulsion containing dapsone for topical administration: a study of in vitro release and epidermal permeation
}

This article was published in the following Dove Press journal:

International Journal of Nanomedicine

4 February 2013

Number of times this article has been viewed

Vinícius Raphael de Almeida

Borges

Alice Simon

Adrian Ricardo Cuello Sena

Lúcio Mendes Cabral

Valéria Pereira de Sousa

Department of Pharmaceutics, Faculty of Pharmacy, Federal University of Rio de Janeiro, Rio de Janeiro, Brazil
Correspondence: Valéria Pereira de Sousa

Department of Pharmaceutics, Faculty of Pharmacy, Federal University of Rio de Janeiro, Av Carlos Chagas Filho, 373, CCS, Bss, Sala 15, Rio de Janeiro,

RJ, 2194I-902, Brazil

Tel +55 2I 25647380

$\mathrm{Fax}+552125647380$

Email valeria@pharma.ufrj.br
Background: Topical administration of dapsone can be an alternative route for treatment of leprosy and can also provide new therapeutic applications for an established drug. However, the physicochemical properties of dapsone make it difficult to incorporate into conventional formulations. The current study was directed toward developing a stable nanoemulsion that contains dapsone which can be adapted for topical use.

Methods: Nanoemulsions were prepared using isopropyl myristate or n-methyl-pyrrolidone as the oil phase, and characterized according to their mean droplet size, conductivity, refractive index, $\mathrm{pH}$, drug content, and stability. The in vitro release of dapsone and its ability to permeate the epidermis were also evaluated.

Results: Physicochemical characterization demonstrated that nanosystems were formed, which had a uniform droplet distribution and a $\mathrm{pH}$ compatible with the skin surface. Use of n-methyl-pyrrolidone provided a greater nanoemulsion region and higher solubilization of dapsone, and increased the in vitro release rate when compared with a nanoemulsion prepared using isopropyl myristate. However, use of isopropyl myristate promoted an increase in in vitro epidermal permeation that followed the Higuchi model. This demonstrates the ability of a nanosystem to influence permeation of dapsone through the skin barrier. Furthermore, the nanoemulsions developed and evaluated here had ideal physicochemical stability over a 3-month period.

Conclusion: Incorporation of dapsone into a nanoemulsion may be a promising system for enabling topical delivery of dapsone, while minimizing skin permeation, for the treatment of acne. The method developed here used isopropyl myristate as the oil phase, and promoted permeation of dapsone through the skin barrier for the treatment of leprosy upon use of n-methylpyrrolidone as the oil phase.

Keywords: dapsone, nanoemulsion, topical, release, permeation

\section{Introduction}

Dapsone (4,4'-diaminodiphenylsulfone) is a drug of the sulfone class and was discovered in $1908 .{ }^{1} \mathrm{~A}$ unique property of dapsone is that it has dual therapeutic activity and demonstrates antimicrobial and anti-inflammatory properties. Since 1943, it has been the drug of choice in the treatment of leprosy because it has bacteriostatic action against Mycobacterium leprae by inhibiting folic acid production via competition with p-aminobenzoate for the active site of dihydropteroate synthase. ${ }^{2}$ Many studies have explored the anti-inflammatory activity of dapsone in diseases characterized by abnormal accumulation of neutrophils. It has obvious application in the field of dermatology, and may ease symptoms in diseases such as acne, Behçet's disease, 
epidermolysis bullosa acquisita, dermatitis herpetiformis, Kaposi's sarcoma, and systemic lupus erythematosus., ${ }^{1,3}$

Despite its obvious therapeutic efficacy in skin diseases, clinical use of dapsone is limited due to its physicochemical properties. Dapsone is a drug with low solubility in water and is categorized as a Class II agent according to the biopharmaceutical classification system proposed by Amidon et al. ${ }^{4}$ This characteristic is unfavorable for the production of dapsone formulations with adequate bioavailability. Our research group has proposed the use of a new oral nanoemulsion system which demonstrates adequate intestinal permeability. ${ }^{5}$ However, oral administration of dapsone is associated with several adverse effects, including hemolytic anemia, peripheral neuropathy, nausea, and headache. These side effects diminish its feasibility for treating skin diseases by the oral route. Many of the adverse effects of dapsone are related to the production of metabolites. In the liver, dapsone is acetylated by $\mathrm{N}$-acetyltransferase which produces monoacetyl dapsone, and upon enzymatic hydroxylation, dapsone hydroxylamine is produced, which is primarily responsible for the development of adverse effects. ${ }^{6}$

Because of the therapeutic relevance of dapsone, it is desirable to reduce its adverse effects using nanotechnology. 7,8 Nanosystems containing dapsone, such as liposomes ${ }^{9}$ and nanoparticles, ${ }^{10}$ have been proposed in the past. An unexplored therapeutic approach for administration of dapsone is the topical route. Utilization of topical applications provides an efficient strategy both for the alternative treatment of leprosy, where the pathogen and lesions are in the skin, and other dermatological indications, such as acne. Among the various colloidal nanosystems available, nanoemulsions are notable because they offer recognized advantages by increasing the solubility of hydrophobic drugs such as dapsone. ${ }^{11}$ Nanoemulsions can be administered by various routes, but topical application has gained prominence due to its advantages. ${ }^{12}$ Nanoemulsions are thermodynamically stable compared with traditional formulations such as creams and ointments, and also have a malleable nanostructure ideal for skin permeation. Further, nanoemulsion systems form spontaneously, which facilitates industrial production and scaling. ${ }^{13-15}$

In this context, the objective of this work was to optimize nanoemulsion systems that effectively incorporate dapsone and provide an ideal dapsone delivery system for topical administration. The nanoemulsions were characterized and their in vitro drug release kinetics were determined. In vitro drug permeation was also evaluated using excised porcine ear epidermis to monitor the performance of the newly developed nanoemulsions.

\section{Materials and methods Materials}

Dapsone was obtained from G Amphray Laboratories (Mumbai, India) and used in the production of the nanoemulsions, and dapsone USP standard, lot 02/572 A (Rockville, MD, USA) was used in all quantitative analyses. Sodium chloride, potassium phosphate monobasic, sodium lauryl sulfate, and isopropyl myristate were obtained from Vetec (Rio de Janeiro, Brazil). Chromatography grade methanol came from Tedia Company Inc (Fairfield, OH, USA). Isobutanol, polysorbate 80 (Tween ${ }^{\circledR} 80$ ), and sorbitan monolaurate 20 (Span ${ }^{\circledR} 20$ ) were obtained from Sigma Chemical Company (St Louis, MO, USA). The n-methyl-pyrrolidone came from Novaire (São Paulo, Brazil). Water used in the experiments was obtained using a Milli-Q gradient A-10 water purification system (Millipore, Bedford, MA, USA).

\section{Construction of pseudoternary phase diagram}

The surfactants Tween 80 and Span 20 (1:1) were homogenized, and then the oil phase, composed of a n-methyl pyrrolidone and isobutanol mixture (oil to cosurfactant, 8:1) was added to the solution. Subsequently, the pseudoternary phase diagram was obtained using the aqueous titration method at room temperature. Mixtures that contained the surfactant and oil phase were prepared in predefined ratios (10:90, 20:80, 30:70, 40:60, $50: 50,60: 40,70: 30,80: 20$, and 90:10). Each of these proportions were titrated by sequentially adding $10 \mu \mathrm{L}$ of water until a visually heterogeneous system had formed. This process was carried out in triplicate. Compositions of mixtures that formed clear and isotropic systems were calculated. ${ }^{16}$ The nanoemulsion region was represented graphically using the Chemix School version 2.50 software (Arne Standnes, Norway).

\section{Development and characterization of nanoemulsions}

The concentration of dapsone incorporated into the nanoemulsion and the amount of oil present in the oil phase (oil + cosurfactant) were refined to optimize the solubility of dapsone. The nanoemulsion systems A-D were assessed to determine the solubility of dapsone in the oil phase by adding excess dapsone $(5 \mathrm{~mL})$. After vigorous stirring for 24 hours, the solution was centrifuged. After dilution, the solution was filtered through a $0.45 \mu \mathrm{m}$ filter and analyzed by high-pressure liquid chromatography.

The nanoemulsion systems were prepared according to the method described by Nandi et al. ${ }^{16}$ Based on the 
pseudoternary diagram, four formulations were prepared using magnetic stirring as a source of agitation for a moderate period of time (Table 1). Two drug incorporation methods were tested in order to obtain a larger degree of drug incorporation in the nanoemulsions and thus more efficient incorporation of dapsone into the formulation. Subsequently, in one dapsone incorporation method, dapsone powder was mixed into the finished formulation, ie, after homogenization of the excipients. In the other method, dapsone was incorporated during preparation of the nanoemulsions, into the oil phase after adding the surfactant, cosurfactant, and oil (oil phase) before finally adding the water. Structural characteristics of the nanoemulsions were evaluated by a electrical conductivity test using a conductivimeter (Mettler Toledo model MPC 227, Columbus, OH, USA), while the refractive index was obtained using an Analytik Jena refractometer (Jena, Germany). The $\mathrm{pH}$ value was measured by immersing the electrode of a Digimed model DM21 (São Paulo, Brazil) into formulations maintained at room temperature. The mean diameter of the internal droplets in the formulations was determined by dynamic light scattering using a particle size analyzer (Horiba model LB-550, Kyoto, Japan), which reports the uniformity of the droplet diameter distribution using the SPAN index. ${ }^{17}$

\section{High performance liquid chromatographic analysis}

The quantification method used to assess incorporation of dapsone into the formulation and its release and permeation properties was developed using high performance liquid chromatography (pump L-2130, column oven L-2300, autosampler L-2200 and diode array detector L-2455, MerckHitachi, Darmstadt, Germany). Chromatographic separation was performed using a Kromasil $\mathrm{C}_{8}$ column $(150 \times 4.6 \mathrm{~mm}$, $5 \mathrm{~mm}$ ) and the mobile phase consisted of a mixture of $30 \mathrm{mM}$ phosphate buffer $\mathrm{pH} 7.4$ and methanol (70:30). The elution was isocratic with a flow rate of $1 \mathrm{~mL}$ per minute and ultraviolet detection at $295 \mathrm{~nm}$.

Table I Composition of the nanoemulsions A, B, C, and D

\begin{tabular}{|c|c|c|c|c|c|c|}
\hline \multirow[t]{3}{*}{$\overline{\text { NE }}$} & \multicolumn{5}{|c|}{ Excipients (\%; w/w) } & \multirow[t]{3}{*}{ Water } \\
\hline & \multicolumn{3}{|c|}{ Oil phase } & \multicolumn{2}{|c|}{ Surfactants } & \\
\hline & IPM & NMP & ISO & T80 & $\mathbf{S 2 0}$ & \\
\hline A & 36.95 & - & 4.60 & 20.90 & 20.90 & 16.65 \\
\hline B & - & 36.95 & 4.60 & 20.90 & 20.90 & 16.65 \\
\hline$C$ & - & 27.33 & 3.87 & 20.90 & 20.90 & 27.00 \\
\hline $\mathrm{D}$ & - & 15.14 & 1.89 & 33.16 & 33.16 & 16.65 \\
\hline
\end{tabular}

Abbreviations: IPM, isopropyl myristate; NMP, N-methyl pyrrolidone; ISO, isobutanol; T80, Tween 80; S20, Span 20; NE, nanoemulsions.
The method was validated by evaluating specificity, linearity, repeatability, intermediate precision, accuracy, and quantification and detection limits. ${ }^{18,19}$ Selectivity properties were evaluated using the Lachrom software provided for the detector and the ratiogram produced, which showed superposition of ultraviolet spectra at different chromatographic peak times. The chosen method should be constant, close to zero, and yield a value that is large enough to be differentiated from noise.

Linearity was tested using three standard dapsone curves constructed using five concentrations in the range of 5 to $100 \mu \mathrm{g} / \mathrm{mL}$. The results were analyzed statistically using linear regression and the least squares method. Precision was determined by evaluating repeatability, while the intermediate precision of the measurements was tested using standards with concentrations of $80 \%, 100 \%$, and $120 \%(20,25$, and $30 \mu \mathrm{g} / \mathrm{mL}$ ), with three replicates each. The relative standard deviation was calculated and a value obtained below $2 \%$ was considered acceptable. Accuracy was evaluated by analyzing three replicates of three independently prepared samples (spike placebo) at concentrations of $80 \%, 100 \%$, and $120 \%$ of the working concentration. Values obtained between $98 \%$ and $102 \%$ of recovery were considered acceptable. The quantification and detection limits were estimated based on the standard deviation of the mean of the y-intercepts obtained from three regression lines which were multiplied by three and ten, respectively, and divided by the slope.

\section{In vitro release and permeation study}

The release and permeation studies were carried out in Franz diffusion cells (Hanson Research, Chatsworth, LA, USA) with an effective diffusion area of $1.767 \mathrm{~cm}^{2}$ and contained $7 \mathrm{~mL}$ of the receptor medium. For the release and permeation studies, the cellulose acetate membranes and porcine ear epidermis were carefully placed at the interface between the donor and receptor compartments, respectively. The young white porcine ears were obtained from a local slaughterhouse supervised by the Institute of Agricultural Protection and Forestry of the Espírito Santo State in Brazil. The epidermal layer was excised and subsequently washed and visually inspected to ensure its integrity. ${ }^{20}$ The segments of epidermis were wrapped in filter paper moistened with saline solution and packed in aluminum foil for storage in a freezer at $-4^{\circ} \mathrm{C}$ for a maximum of 60 days. ${ }^{21}$ The frozen epidermis was rehydrated by incubation in a saline solution at room temperature for one hour before being placed in the Franz cells. In each experiment, the porcine skin segments were obtained from different animals. 
The mixture containing $0.1 \mathrm{M}$ potassium phosphate buffer $\mathrm{pH} 7.4$ and $2.0 \%(\mathrm{w} / \mathrm{w})$ Tween 80 was used as the receptor medium. The Franz cells were maintained at a constant temperature of $37^{\circ} \mathrm{C} \pm 0.5^{\circ} \mathrm{C}$ during the experiment, while being stirred on a magnetic stirrer at $350 \mathrm{rpm}$. The donor compartment was filled with $400 \mu \mathrm{L}$ of the formulation being tested. Subsequently, $1 \mathrm{~mL}$ samples were collected from the receptor compartment at one, 2, 3, 4, 5, 6, 7, 8, 20,23 , and 24 hours after starting the analysis and replaced with the same volume of receptor medium. All samples were analyzed by high performance liquid chromatography. The cumulative amount of dapsone released or that permeated through the membrane per area of diffusion was plotted as a function of time.

\section{Evaluation of release and permeation kinetics}

The release and permeation kinetics were determined by linear regression analysis of the in vitro release and permeation curves in four models, ie, zero order, Higuchi, first order, and Korsmeyer-Peppas using the Statistica 7 software package (Statsoft Company, Tulsa, OK, USA). ${ }^{22}$ The mathematical model that best expressed the kinetic release profile was selected based on the highest coefficient of determination $\left(\mathrm{R}^{2}\right)$.

The cumulative amount of dapsone released and permeated was calculated using the exposed area $\left(\mu \mathrm{g} / \mathrm{cm}^{2}\right)$, and the results were plotted as a function of time (hours) and are shown as the mean \pm standard deviation of six experiments for each nanoemulsion. From the plot, the flux of dapsone across the membrane $(J)$ was calculated from the linear portion of the slope and expressed as $\mu \mathrm{g} / \mathrm{cm}^{2} /$ hour.

\section{Statistical analysis}

All data are shown as the mean \pm standard deviation. Statistical analysis was performed using the one-way analysis of variance test (Tukey multiple comparisons) with GraphPad Prism $^{\circledR} 5.0$ software (La Jolla, CA, USA). Values obtained below $P<0.05$ were considered to be statistically significant.

\section{Stability test}

Two batches of selected formulations were bottled in amber glass containers capped with a rubber stopper to perform the stability study. The test was carried out in a climatic chamber (Nova Ética 420/CLDTS 300 model, São Paulo, Brazil) at a temperature of $25^{\circ} \mathrm{C} \pm 2{ }^{\circ} \mathrm{C}$ and relative humidity of $65 \% \pm 5 \%$ over a 6 -month period. The samples were characterized according to their visual properties, droplet size, and electrical conductivity after zero and 6-month time points.

\section{Results and discussion}

In this study, the nanoemulsion system selected as a vehicle for dapsone was proposed by Nandi et $\mathrm{al},{ }^{16}$ and uses isopropyl myristate as the oil phase (Table 1, nanoemulsion A). Using this system, it was possible to solubilize up to $2 \%(\mathrm{w} / \mathrm{w})$ of dapsone. Higher concentrations of dapsone, up to $3.5 \%$, could be incorporated, but visual stability was not maintained in the nanoemulsion at this concentration during the 60-day test period under ambient conditions. Because of the difficulties in solubilizing dapsone, two possible strategies were tested for incorporating the drug into the system, ie, addition of the drug into the oil phase prior to addition of the aqueous phase, and addition of the drug to the finished formulation. When using dapsone $2.5 \%$, it was only possible to obtain a stable system when dapsone was incorporated into the finished nanoemulsion formulation. However, when $2 \%$ dapsone was used, the system remained stable for 60 days for either incorporation procedure tested. Furthermore, the solubility of dapsone in the nanoemulsion A oil phase was $5.81 \% \pm 0.16 \%(\mathrm{w} / \mathrm{w})$.

In order to maximize incorporation of dapsone into the nanosystem, an alternative composition of the oil phase was proposed, whereby n-methyl pyrrolidone replaced isopropyl myristate (Table 1, nanoemulsion B). This modification was based on the finding that dapsone had increased solubility in the nanoemulsion B oil phase $(26.8 \% \pm 0.47 \%$, w/w), which promoted incorporation of dapsone $5 \%(\mathrm{w} / \mathrm{w})$. Although higher concentrations of dapsone may be incorporated into this system, this concentration was selected because of efficacy and safety considerations in in vivo studies. ${ }^{23}$ Further, n-methyl-pyrrolidone is used as a solubilizing agent in pharmaceutical formulations for oral and parenteral use and as a penetration enhancer for lipophilic drugs. ${ }^{24}$ These characteristics make n-methyl-pyrrolidone an ideal candidate, and recently they have been exploited in applications testing the efficacy of n-methyl-pyrrolidone in transdermal nanosystems containing granisetron hydrochloride, ${ }^{25}$ and in formulations designed for topical use, such as lidocaine, ${ }^{26}$ estradiol,,$^{27}$ bupranolol, ${ }^{28}$ and insulin..$^{29}$ In formulations intended for topical administration, the aqueous phase and surfactants affect skin permeability by hydration of the stratum corneum and breakdown of the lipoprotein matrix, resulting in decreased resistance to drug diffusion. ${ }^{30-32}$ As shown in Figure 1, the pseudoternary phase diagram of the 


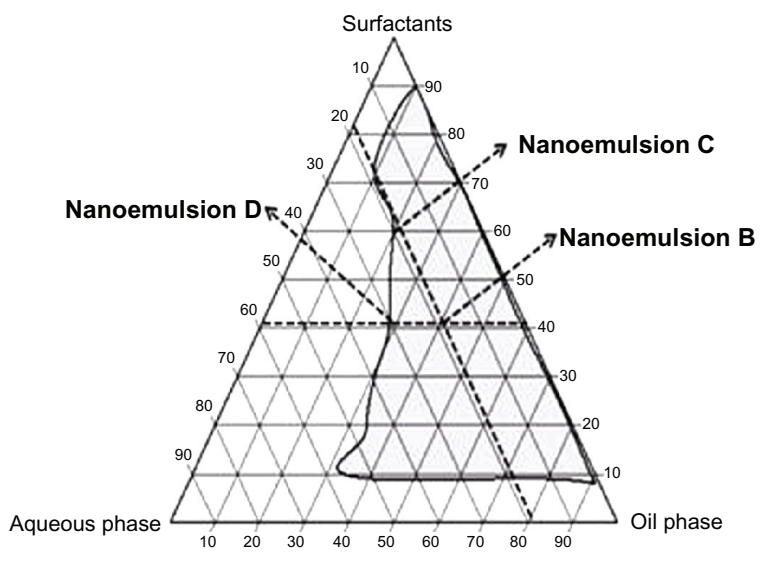

Figure I Pseudoternary phase diagram of systems containing water/N-methyl pyrrolidone and isobutanol/Tween ${ }^{\circledR} 80$ and $\operatorname{Span}^{\circledR} 20$.

Note: The area in gray indicates a nanoemulsion. Nanoemulsions B, C, and D are indicated.

n-methyl-pyrrolidone-containing system shows a large nanoemulsion region. According to this diagram, two new formulations (nanoemulsions $\mathrm{C}$ and $\mathrm{D}$ ) were proposed based upon verification that the proportion of aqueous phase to surfactant is a factor influencing skin permeability. In this respect, formulation $\mathrm{C}$ contains the maximal proportion of aqueous phase, while maintaining the same quantity of surfactant in relation to formulation B. Meanwhile, formulation D shows the maximal proportion of surfactant, but having the same quantity of aqueous phase in relation to formulation B. The solubility of dapsone in the oil phase of nanoemulsions $\mathrm{C}$ and D was not significantly different from the solubility in the oil phase of nanoemulsion B. The formulations shown in Table 1 appeared to be isotropic systems because they were clear, translucent, and transparent. ${ }^{33,34}$

Table 2 shows the nanoemulsion droplet size, which is an important parameter for demonstrating that the internal phase of the systems are formed with a nanoscale diameter, and a polydispersity index lower than 1.0. In addition, the nanoemulsion had a uniform distribution of internal phase droplet sizes. This indicates that additional techniques such as ultrasound, which is normally employed to increase uniformity of the droplet size distribution, can be dispensed with. Thus, this modification could reduce the production costs for the system. ${ }^{17,35}$

The formulations that contained n-methyl-pyrrolidone in the oil phase (nanoemulsions B, C, and D) allowed solubilization of dapsone $5 \%(\mathrm{w} / \mathrm{w})$, while maintaining visual stability during 60 days of storage for the two strategies used for incorporation of the drug, while nanoemulsion A showed incorporation of only $2 \%(\mathrm{w} / \mathrm{w})$ dapsone. This can be explained by the physicochemical characteristics of
Table 2 Evaluation of the mean droplet size in the nanoemulsions $A, B, C$ and $D$, in the absence of drug (control) and in the presence of the drug incorporated in the oil phase (OP) and in the finished formulation (FF)

\begin{tabular}{lllcl}
\hline NE & $\begin{array}{l}\text { Drug } \\
\text { incorporation } \\
\text { method }\end{array}$ & $\begin{array}{l}\text { DAP } \\
\text { incorporated } \\
(\%)\end{array}$ & $\begin{array}{l}\text { Mean droplet } \\
\text { size (nm) }\end{array}$ & $\begin{array}{l}\text { SPAN } \\
\mathbf{2 0}\end{array}$ \\
\hline A & Control & - & $9.3 \pm 2.2$ & 0.6167 \\
& OP & 2.0 & $9.6 \pm 2.5$ & 0.6765 \\
& FF & 2.0 & $11.9 \pm 2.3$ & 0.5068 \\
B & Control & - & $9.0 \pm 2.3$ & 0.6531 \\
& OP & 5.0 & $9.0 \pm 2.30$ & 0.6531 \\
& FF & 5.0 & $8.9 \pm 2.3$ & 0.7089 \\
C & Control & - & $11.2 \pm 2.1$ & 0.4801 \\
& OP & 5.0 & $7.5 \pm 1.7$ & 0.6245 \\
& FF & 5.0 & $9.9 \pm 2.7$ & 0.7195 \\
D & Control & - & $10.0 \pm 2.3$ & 0.5998 \\
& OP & 5.0 & $9.0 \pm 2.8$ & 0.8288 \\
& FF & 5.0 & $8.7 \pm 2.4$ & 0.7194 \\
\hline
\end{tabular}

Notes: Mean \pm standard deviation; $\mathrm{n}=3$.

Abbreviations: DAP, dapsone; NE, nanoemulsion.

n-methyl-pyrrolidone, which contains a nonpolar carbon that can weaken the structure of hydrogen bonding in water, allowing it to act also as a cosolvent, thereby increasing drug solubilization. ${ }^{24}$ Incorporation of the drug in the formulations did not alter the nanometric size, with a uniform distribution of droplet sizes being maintained (Table 2).

Table 3 shows the physicochemical characteristics of the study formulations and verifies the structural changes resulting from the different incorporation processes. The $\mathrm{pH}$ values for the nanoemulsions are compatible with those of the skin, ie, approximately 5.4-6.9, and are suitable for topical administration. Further, considering that dapsone has a pKa of 1.0, the drug is nonionized at this $\mathrm{pH}$, so is ideal for permeation thorough the stratum corneum. ${ }^{36}$ Results of the physicochemical characterization of the nanoemulsions, along with their refractive index and electrical conductivity, indicate that no structural changes occurred due to the different methods used for drug incorporation.

In this study, a method was developed and validated for quantification of dapsone in the proposed pharmaceutical formulations using high performance liquid chromatography which examined the properties of release and permeation. This method was developed from the process described by Queiroz et al, ${ }^{37}$ which provided specificity for the dapsone assay in nanoemulsions. From an average of three dapsone standard curves in the range of $5-100 \mu \mathrm{g} / \mathrm{mL}$, a linear coefficient of 0.9999 was obtained, along with a slope of $467895.5 \pm 3916.6$ and a y-intercept value of $53425.8 \pm 68954.5$. The detection limits and quantification 
Table 3 Physicochemical properties of nanoemulsions A, B, C, and D in the absence and presence of the drug incorporated into the oil phase (OP) and in the finished formulation (FF)

\begin{tabular}{|c|c|c|c|c|c|}
\hline NE & Drug incorporation method & Conductivity & Refractive index & $\mathrm{pH}$ & Drug content (\%) \\
\hline \multirow[t]{3}{*}{$A$} & Control & $0.63 \pm 0.05$ & $\mathrm{I} .432 \pm 0.00 \mathrm{I}$ & $5.65 \pm 0.05$ & - \\
\hline & OP & $0.08 \pm 0.2$ & $\mathrm{I} .437 \pm 0.00 \mathrm{I}$ & $5.42 \pm 0.01$ & $|00.7| \pm 0.94$ \\
\hline & $\mathrm{FF}$ & $0.05 \pm 0.03$ & $1.438 \pm 0.000$ & $5.63 \pm 0.10$ & $99.39 \pm 0.98$ \\
\hline \multirow[t]{3}{*}{ B } & Control & $17.52 \pm 0.94$ & $\mathrm{I} .452 \pm 0.00 \mathrm{I}$ & $6.78 \pm 0.07$ & - \\
\hline & OP & $15.20 \pm 1.70$ & $1.495 \pm 0.003$ & $6.78 \pm 0.02$ & $101.07 \pm 0.84$ \\
\hline & $\mathrm{FF}$ & $15.35 \pm 2.22$ & $1.459 \pm 0.003$ & $6.89 \pm 0.06$ & $98.11 \pm 0.92$ \\
\hline \multirow[t]{3}{*}{ C } & Control & $34.87 \pm 1.10$ & $\mathrm{I} .437 \pm 0.00 \mathrm{I}$ & $6.18 \pm 0.00$ & - \\
\hline & OP & $28.43 \pm 3.01$ & $\mathrm{I} .446 \pm 0.00 \mathrm{I}$ & $6.10 \pm 0.03$ & $100.86 \pm 0.33$ \\
\hline & $\mathrm{FF}$ & $26.67 \pm 0.59$ & $\mathrm{I} .445 \pm 0.003$ & $6.07 \pm 0.04$ & $98.23 \pm 1.29$ \\
\hline \multirow[t]{7}{*}{ D } & Control & $1 \mathrm{I} .73 \pm 0.63$ & $\mathrm{I} .45 \mathrm{I} \pm 0.00 \mathrm{I}$ & $6.22 \pm 0.04$ & - \\
\hline & OP & $11.63 \pm 1.79$ & $1.459 \pm 0.004$ & $6.18 \pm 0.04$ & $100.67 \pm 1.49$ \\
\hline & $\mathrm{FF}$ & $11.23 \pm 1.70$ & $1.460 \pm 0.002$ & $6.19 \pm 0.03$ & $103.21 \pm 1.33$ \\
\hline & Excipients & & & & \\
\hline & Water & $2.85 \pm 0.23$ & $\mathrm{I} .328 \pm 0.00 \mathrm{I}$ & $5.69 \pm 0.01$ & - \\
\hline & Isopropyl myristate & $0.00 \pm 0.00$ & $1.434 \pm 0.002$ & $4.60 \pm 0.03$ & - \\
\hline & n-methyl pyrrolidone & $1.40 \pm 0.31$ & $1.415 \pm 0.001$ & $9.40 \pm 0.01$ & - \\
\hline
\end{tabular}

Notes: Mean \pm standard deviation; $\mathrm{n}=3$.

Abbreviation: NE, nanoemulsion.

values were calculated as 0.44 and $1.47 \mu \mathrm{g} / \mathrm{mL}$, respectively, and are considered satisfactory for the intended purpose. Table 4 shows that the relative standard deviation was above $1 \%$, confirming that this method is accurate. ${ }^{18,19}$ The recovery values obtained were in the range of $98 \%-102 \%$ at all three levels evaluated. ${ }^{18,19}$ The validation data showed that the high performance liquid chromatography method is suitable for determination of the amount of dapsone present in the nanoemulsions developed in this study, and was also valid for drug quantification in the receptor medium used for the release and permeation studies. The amount of drug diluted in the receptor medium did not affect the results in these studies.

Figure 2 shows the dapsone release profiles over a 24-hour period, and the values obtained are shown in Table 5. All release profiles followed Higuchi's model for dapsone release (Table 5). This model, based on Fick's law, indicates that

Table 4 Evaluation of intraday and interday precision and accuracy of the quantification method of dapsone

\begin{tabular}{|c|c|c|c|c|}
\hline \multirow[t]{2}{*}{ Level } & \multicolumn{2}{|l|}{ Precision } & \multicolumn{2}{|l|}{ Accuracy } \\
\hline & $\begin{array}{l}\text { Intraday } \\
\text { RSD (\%) }\end{array}$ & $\begin{array}{l}\text { Interday } \\
\text { RSD (\%) }\end{array}$ & $\begin{array}{l}\text { Recovery } \\
\text { (\%) }\end{array}$ & $\begin{array}{l}\text { RSD } \\
\text { (\%) }\end{array}$ \\
\hline \multirow[t]{2}{*}{ Low } & 0.56 & 0.66 & 99.60 & 0.47 \\
\hline & 0.47 & & & \\
\hline \multirow[t]{2}{*}{ Medium } & 0.75 & 1.20 & 99.01 & 0.40 \\
\hline & 0.37 & & & \\
\hline \multirow[t]{2}{*}{ High } & 0.40 & 0.85 & 101.03 & 0.75 \\
\hline & 0.16 & & & \\
\hline
\end{tabular}

Abbreviation: RSD, relative standard deviation. the formulation plays an important role in the process of drug release into the receptor medium. ${ }^{22}$ The formulations containing n-methyl-pyrrolidone, ie, nanoemulsions B, C, and $\mathrm{D}(\mathrm{OP}$ or $\mathrm{FF}$ ), provided higher flux $(J)$ of dapsone in relation to nanoemulsion $\mathrm{A}$ ( $\mathrm{OP}$ or $\mathrm{FF}$ ), which contained
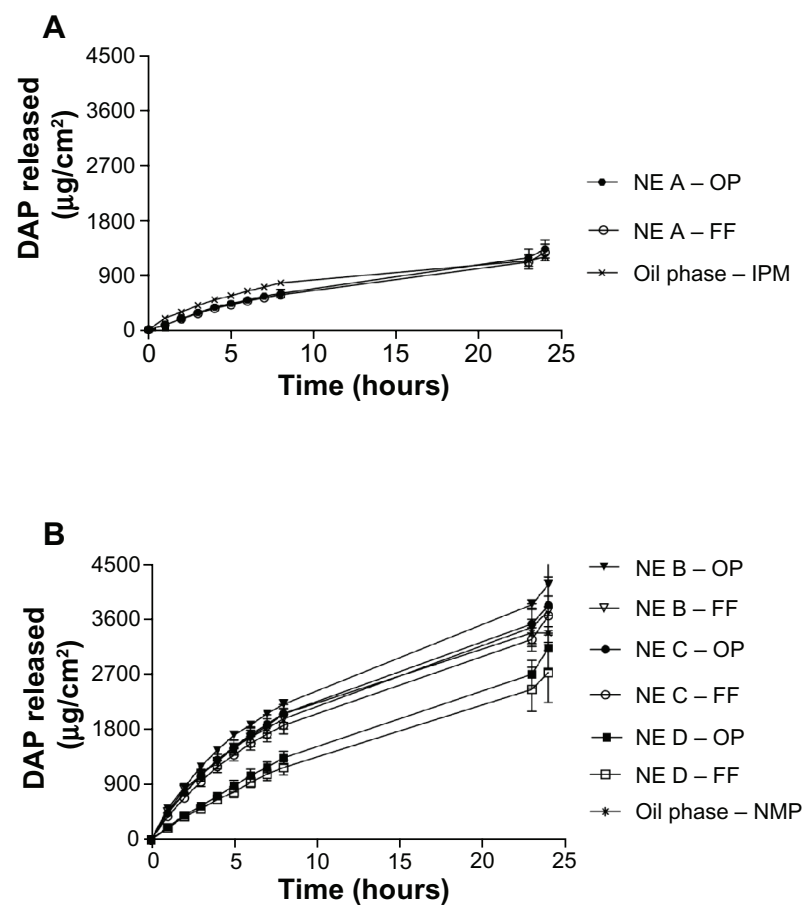

Figure 2 Dapsone release profile from nanoemulsion $A(A)$ and nanoemulsions B, $C$, and $\mathrm{D}(\mathrm{B})$ with the drug incorporated in the oil phase (OP) and in the finished formulation (FF).

Abbreviation: DAP, dapsone; NE, nanoemulsion; IPM, isopropyl myristate; NMP, n-methyl-pyrrolidone; OP, oil phase; FF, finished formulation. 
Table 5 Evaluation of in vitro release rate of DAP nanoemulsions

\begin{tabular}{|c|c|c|c|c|c|}
\hline NE & & Flux & Lag time & Drug released $^{a}$ & Linear \\
\hline A & OP & $60.74 \pm 4.91^{\mathrm{e}}$ & $30.08 \pm 12.89$ & $1326.06 \pm 146.32$ & 0.9984 \\
\hline & $\mathrm{FF}$ & $59.74 \pm 7.62$ & $31.10 \pm 11.72$ & $1270.64 \pm 137.05$ & 0.9994 \\
\hline B & OP & $201.62 \pm 4.36$ & $10.58 \pm 2.45$ & $4176.05 \pm 356.93$ & 0.9978 \\
\hline & $\mathrm{FF}$ & $181.03 \pm 16.16$ & $13.25 \pm 4.16$ & $3747.92 \pm 553.95$ & 0.9986 \\
\hline C & OP & $199.64 \pm 16.19$ & $19.49 \pm 6.83$ & $3837.34 \pm 410.87$ & 0.9999 \\
\hline & $\mathrm{FF}$ & $182.52 \pm 13.93$ & $20.18 \pm 7.63$ & $3668.41 \pm 319.44$ & 0.9989 \\
\hline D & OP & $159.29 \pm 11.13^{f}$ & $58.81 \pm 11.89$ & $3140.12 \pm 350.94$ & 0.9975 \\
\hline & $\mathrm{FF}$ & $136.75 \pm 17.78$ & $53.02 \pm 14.74$ & $2738.88 \pm 492.51$ & 0.9973 \\
\hline Oil & & $84.92 \pm 21.0 \mathrm{I}^{\mathrm{c}}$ & $14.66 \pm 7.52$ & $1196.76 \pm 44.44$ & 0.9992 \\
\hline Oil & & $197.45 \pm 6.46^{d}$ & $6.42 \pm 2.44$ & $3386.05 \pm 286.78$ & 0.9993 \\
\hline
\end{tabular}

Notes: Mean \pm standard deviation, $\mathrm{n}=6$. ${ }^{\mathrm{a}}$ Drug released after $24 \mathrm{~h}$ of experiment. All formulations followed Higuchi's model. Statistical analysis of the flux $(J)$ : one-way ANOVA (multiple comparisons Tukey's test). ${ }^{b}$ The drug incorporation method was statistically similar for each NE ( $\left.P>0.05\right)$; ' 0 il phase-IPM was statistically similar $(P>0.05)$ compared to NE A; 'oil phase-NMP was statistically similar $(P>0.05)$ compared to NE B and C and statistically different $(P<0.00 \mathrm{I})$ compared to NE D; ${ }^{\text {eNE }} \mathrm{A}$ was statistically different $(P<0.00 \mathrm{I})$ compared to $\mathrm{NE} B, C$ and $D ;{ }^{f} \mathrm{NE} D$ was statistically different $(P<0.00 \mathrm{I})$ compared to NE B and C.

Abbreviations: DAP, dapsone; NE, nanoemulsion; IPM, isopropyl myristate; NMP, n-methyk-pyrrolidone; OP, oil phase; FF, finished formulation.

isopropyl myristate in the oil phase. The increased level of solubilization was attributed to n-methyl-pyrrolidone which was shown to be a major factor in increasing in vitro drug release from nanoemulsions $\mathrm{B}, \mathrm{C}$, and $\mathrm{D}$ in relation to nanoemulsion A. This is because of the increased thermodynamic activity resulting from the presence of the drug in the vehicle. Controls were performed for nanoemulsion $A$ and nanoemulsion $\mathrm{B}, \mathrm{C}$, and $\mathrm{D}$ to determine the amount of drug dissolved in isopropyl myristate and n-methyl-pyrrolidone, respectively. The nanoemulsions showed no statistically significant differences in flux compared with the respective controls; however, the controls showed a reduction in lag time.

Because the quantity of the aqueous phase can influence release of dapsone, this parameter was assessed in the nanoemulsions containing n-methyl-pyrrolidone. As shown in Figure 2 and Table 5, the amount of aqueous phase, ie, $27.0 \%$ in nanoemulsion $\mathrm{C}$ compared with $16.9 \%$ in nanoemulsion $\mathrm{B}$, did not significantly alter the release profiles. However, the lag time was shorter in the formulation containing a reduced amount of water, ie, nanoemulsion B, which showed a time lag of about 10-13 minutes, whereas nanoemulsion $\mathrm{C}$ had a lag time of 19-20 minutes.

The effect on drug release of varying the amount of surfactant was also studied. ${ }^{31}$ The amount of surfactant in nanoemulsion $\mathrm{D}$ corresponds to $66.3 \%$ of the total formulation, while in nanoemulsions $\mathrm{B}$ and $\mathrm{C}$ it corresponds to $41.8 \%$. Despite the increased amount of surfactant present in nanoemulsion $\mathrm{D}$, the physicochemical properties remained unaltered (Table 3), providing a lower flux and increasing the lag time for dapsone release compared with nanoemulsions $\mathrm{B}$ and $\mathrm{C}$ (Table 5), and indicating that lag time influences the ability to release dapsone. The flux and amount of drug released from nanoemulsion B and C were significantly different from the control (oil phase, n-methyl-pyrrolidone). As shown in Figure 2, there were no significant changes in the release profiles $(P>0.05)$ for the same formulation resulting from a change in drug incorporation methods. This suggests that the method of preparation does not affect release of the active drug from the nanoemulsion. Therefore, in vitro permeation was evaluated only in nanoemulsions obtained by incorporating the drug into the oil phase.

As shown in Figure 3 and Table 6, nanoemulsion A achieved the greatest dapsone permeation, ie, $1480.7 \mu \mathrm{g}$ per $\mathrm{cm}^{2}$ of skin over a 24-hour period. All formulations con-

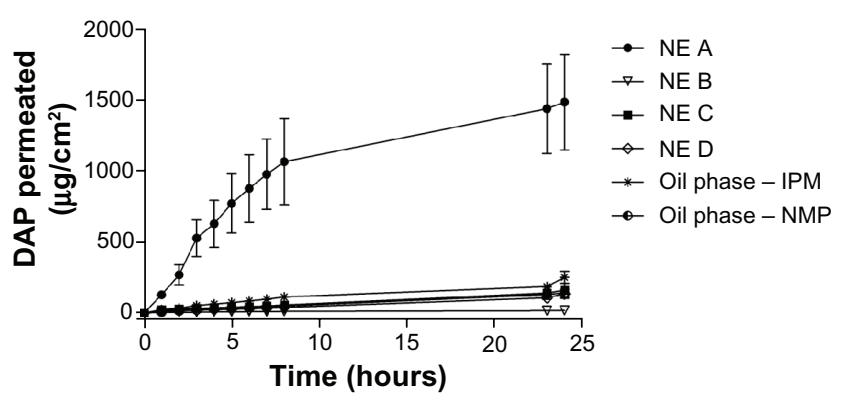

Figure 3 Dapsone permeation profile through porcine epidermis from nanoemulsions $A, B, C$, and D with the drug incorporated in the oil phase in comparison with controls.

Abbreviations: DAP, dapsone; NE, nanoemulsion; IPM, isopropyl myristate; NMP, n-methyl-pyrrolidone. 
Table 6 Evaluation of in vitro permeation rate of DAP nanoemulsions

\begin{tabular}{|c|c|c|c|c|c|}
\hline $\mathrm{NE}^{\mathrm{a}}$ & Flux $\left(J ; \mu \mathrm{g} \cdot \mathrm{cm}^{-2} \cdot \mathbf{h}^{-1}\right)$ & Lag time (h) & $\begin{array}{l}\text { DAP permeated* } \\
\left(\mu \mathrm{g} / \mathrm{cm}^{2}\right)\end{array}$ & $\begin{array}{l}\text { Kinetic } \\
\text { model }\end{array}$ & $\begin{array}{l}\text { Linear } \\
\text { correlation (r) }\end{array}$ \\
\hline A & $109.66 \pm 32.60^{b}$ & $0.42 \pm 0.11$ & $1480.66 \pm 335.30$ & Higuchi & 0.9941 \\
\hline B & $1.02 \pm 0.10$ & $3.86 \pm 0.78$ & $19.76 \pm 4.87$ & Zero order & 0.9858 \\
\hline C & $4.83 \pm 1.39$ & $3.83 \pm 0.19$ & $161.40 \pm 47.39$ & Zero order & 0.9977 \\
\hline D & $3.70 \pm 0.47$ & $2.99 \pm 0.51$ & $135.04 \pm 31.16$ & Zero order & 0.9918 \\
\hline Oil phase - IPM & $11.77 \pm 1.03$ & $0.35 \pm 0.15$ & $251.0 \pm 42.09$ & Higuchi & 0.9982 \\
\hline Oil phase - NMP & $2.62 \pm 0.18^{c}$ & $1.46 \pm 0.48$ & $142.9 \pm 27.87$ & Zero order & 0.9810 \\
\hline
\end{tabular}

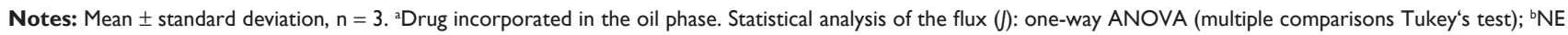
A was statistically different $(P<0.00 \mathrm{I})$ compared to NE B, C, and D and oil phase-IPM; ' coil phase-NMP was statistically similar $(P>0.05)$ compared to NE B, C and D.

Abbreviations: DAP, dapsone; NE, nanoemulsion; IPM, isopropyl myristate; NMP, n-methyl-pyrrolidone.

taining n-methyl-pyrrolidone (nanoemulsions B, C, and D) showed a small flux and a similar amount of permeated drug $(P>0.05)$. These formulations were significantly different $(P<0.001)$ from the formulation containing isopropyl myristate (nanoemulsion A).

Comparing the results for in vitro release with the results for in vitro permeation of dapsone from nanoemulsions $\mathrm{B}$, C, and D, it can be seen that, after 24 hours, these systems were not capable of overcoming the epidermal barrier and achieving satisfactory permeation of dapsone, despite the large amounts of dapsone released by the systems. However, the nanoemulsion A system enabled adequate drug permeation through the skin. Accordingly, nanoemulsion A provided an increased in vitro permeation flux through the epidermis $\left(109.66 \pm 32.60 \mu \mathrm{g} / \mathrm{cm}^{2} /\right.$ hour with a time lag of approximately 25 minutes). The results for nanoemulsion A are significantly different from those for the control (oil phase, isopropyl myristate), which may be related to the characteristics of the oil phase, in which isopropyl myristate was used, because dapsone has a partition coefficient value $(\log \mathrm{P})$ of 1.32 and therefore dissolved preferentially in the oily domain of the nanoemulsion. ${ }^{38}$ It is known that the oily regions of the nanoemulsion can target the drug directly to lipid regions in the epidermis and stratum corneum interlayer by destabilization. ${ }^{39}$ Although the mechanism of permeation observed for isopropyl myristate is unknown, this excipient may have promoted the above-mentioned effects, leading to increased permeation of the drug released by nanoemulsion $\mathrm{A}$. Dapsone remained stable during the release and permeation studies using both systems, with no degradation products identified on chromatographic analysis.

Lipophilic derivatives such as n-methyl-pyrrolidone have shown an ability to penetrate hydrophobic regions of the stratum corneum and reduce its barrier function. ${ }^{40}$ This phenomenon was expected for nanoemulsions B, C, and D. However, the permeation rate obtained for these formulations (close to zero order kinetics) indicates that the excipients used do not interfere with permeation of the drug, and explains the similarity observed in the in vitro permeation profiles of nanoemulsions B, C, and D (Table 6).

Studies of the differences in performance of the formulations pertaining to drug release and permeation can be tailored for use in different clinical applications. The formulations containing n-methyl-pyrrolidone (nanoemulsions B and C) showed a large amount of drug release with low epidermal permeability. Such formulations when applied topically have minimal systemic absorption, rendering them promising for the treatment of superficial skin diseases, eg, acne and dermatitis herpetiformis. Despite its efficacy for treating these types of disease, dapsone has limited use due to its systemic toxicity. Only one gel formulation containing dapsone $5 \%$ is commercially available on the international market. ${ }^{7,23,41}$

However, nanoemulsion A, containing isopropyl myristate, enables a high degree of dapsone permeation across the stratum corneum and could be used effectively in diseases where the site of action of dapsone is localized to the inner skin layers, as in the treatment of leprosy. Considering that the dapsone concentration indicated for bactericidal action against $M$. leprae is approximately $5 \mathrm{mg} / \mathrm{mL}^{34}$ the in vitro flow permeation obtained for nanoemulsion A $\left(109.66 \pm 32.60 \mu \mathrm{g} / \mathrm{cm}^{2} /\right.$ hour $)$ indicates that this formulation has potential clinical efficacy. Therefore, dapsone may be developed as a topical alternative for the treatment of leprosy, which presently needs to be administered orally. Vectorization of dapsone to the etiological agent could achieve a dose reduction and limit any adverse effects.

Two formulations (nanoemulsions A and B) were selected for evaluation of stability. Table 7 shows the results of 6 months of storage for the long-term stability study. The appearance and electrical conductivity of the nanoemulsions remained constant compared with the initial values obtained 
Table 7 Stability study of nanoemulsions A and B containing DAP incorporated into the OP after 180 days of storage at room temperature

\begin{tabular}{lcc}
\hline Test & \multicolumn{2}{l}{ Results after I80 days of storage } \\
\cline { 2 - 3 } & NE A & NE B \\
\hline Appearance & Homogeneous, clear & Homogeneous, clear \\
& and transparent & and transparent \\
Mean droplet size $(\mathrm{nm})$ & $12.1 \pm 1.07$ & $9.8 \pm 0.7 \mathrm{I}$ \\
Conductivity $(\mu \mathrm{S} / \mathrm{cm})$ & $0.06 \pm 0.03$ & $14.42 \pm 0.36$ \\
Drug content $(\%)$ & $93.72 \pm 1.28$ & $96.23 \pm 0.89$ \\
\hline
\end{tabular}

Notes: Mean \pm standard deviation; $\mathrm{n}=3$.

Abbreviations: DAP, dapsone; NE, nanoemulsion; OP, oil phase.

(Table 3), indicating that no phase inversion or structural changes occurred. The mean droplet diameter was measured after the storage period and compared with the initial value, and was found to be similar (Table 2). No agglomeration was observed, so the composition of the formulation appears to be adequate for maintaining the interfacial tension required for nanoemulsion systems.

\section{Conclusion}

In this work, it was found that it is possible to incorporate dapsone into nanoemulsion systems containing excipients that are compatible with skin. The physicochemical characterization performed here demonstrates that the mean droplet size was in the nanometer range, with good uniformity of diameter and a $\mathrm{pH}$ suitable for topical formulation. The use of different oil phases in the nanoemulsion systems enabled evaluation of the differential performance of the systems with regard to in vitro release and permeation of dapsone, with potential for pharmaceutical application. In summary, it was possible to obtain systems which can deliver dapsone topically and promote its permeation across the epidermis.

\section{Acknowledgments}

This work was supported by FAPERJ and CAPES Edital CAPES Nanobiotecnologia. The authors are grateful to Michelle Parvatiyar for her English language review.

\section{Disclosure}

The authors report no conflicts of interest in this work.

\section{References}

1. Sago J, Hall RP. Dapsone. Dermatol Ther. 2002;15:340-351.

2. Paniker U, Levine N. Dapsone and sulfapyridine. Dermatol Clin. 2001;19:79-86.

3. Wakabayashi M, Fujii N, Fujimoto N, Tanaka T. Cystic acne successfully treated with diaminodiphenylsulfone. J Dermatol. 2011;38: 502-504.
4. Amidon GL, Lennernäs H, Shah VP, Crison JR. A theoretical basis for a biopharmaceutic drug classification: the correlation of in vitro drug product dissolution and in vivo bioavailability. Pharm Res. 1995;12: 413-420.

5. Monteiro LM, Lione VF, do Carmo FA, et al. Development and in silico bioavailability studies of dapsone oral nanoemulsion systems. Int $J$ Nanomedicine. 2012;7:5175-5182.

6. Wozel VEG. Innovative use of dapsone. Dermatol Clin. 2010;28: 599-610.

7. Pupe CG, Villardi M, Rodrigues CR, et al. Preparation and evaluation of antimicrobial activity of nanosystems for the control of oral pathogens Streptococcus mutans and Candida albicans. Int J Nanomedicine. 2011;6:2581-2590.

8. Ruenraroengsak P, Cook JM, Florence AT. Nanosystem drug targeting: facing up to complex realities. J Control Release. 2010;141:265-276.

9. Chougule M, Padhi B, Misra A. Development of spray dried liposomal dry powder inhaler of dapsone. AAPS Pharm Sci Tech. 2008;9:47-53.

10. Saluja V, Chopra D, Singh N, Sekhon BS. Anti-inflammatory potential of dapsone loaded chitosan nanoparticles in streptozotocin-induced experimental dementia. IJPSN. 2011;4:1347-1358.

11. Zhang Y, Gao J, Zheng H, Zhang R, Han Y. The preparation of 3,5-dihydroxy-4-isopropulstilbene nanoemulsion and in vitro release. Int J Nanomedicine. 2011;6:649-657.

12. Salim N, Basri M, Rahman MB, Abdullah DK, Basri H. Modification of palm kernel oil esters nanoemulsions with hydrocolloid gum for enhanced topical delivery of ibuprofen. Int $J$ Nanomedicine. 2012;7:4739-4747.

13. Djekic L, Primorac M. The influence of cosurfactants and oils on the formation of pharmaceutical nanoemulsions based on PEG-8 caprylic/ capric glycerides. Int J Pharm. 2008;352:231-239.

14. Elshafeey AH, Kamel AO, Fathallah MM. Utility of nanosized nanoemulsion for transdermal delivery of tolterodine tartrate: ex-vivo permeation and in-vivo pharmacokinetic studies. Pharm Res. 2009;226:2446-2453.

15. Cevc G, Vierl U. Nanotechnology and the transdermal route: a state of the art review and critical appraisal. $J$ Control Release. 2010;141:277-299.

16. Nandi I, Bari M, Joshi H. Study of isopropyl myristate nanoemulsion system containing cyclodextrins to improve the solubility of 2 model hydrophobic drugs. AAPS Pharm Sci Tech. 2003;4:E10.

17. Sarkar A, Ruma Rano, Mishra KK, Sinha IN. Particle size distribution profile of some Indian fly ash - a comparative study to assess their possible uses. Fuel Process Technol. 2005;86:1221-1238.

18. US Pharmacopeia. NF-29, 34th ed. Rockville, MD: US Pharmacopeia Convention; 2011.

19. International Conference on Harmonization. Q2B. Validation of analytical procedures: Methodology. US Department of Health and Human Services Food and Drug Administration, 1996. Available from: http:// www.fda.gov/downloads/Drugs/GuidanceComplianceRegulatory Information/Guidances/ucm073384.pdf. Accessed January 6, 2013.

20. Junyaprasert VB, Boonme P, Songkro S, Krauel K, Rades T. Transdermal delivery of hydrophobic and hydrophilic local anesthetics from o/w and w/o Brij 97-based microemulsion. J Pharm Sci. 2007;10:288-298.

21. Moser K, Kriwet K, Naik A, Kalia YN, Guy RH. Passive skin penetration enhancement and its quantification in vitro. Eur J Pharm Biopharm. 2001;52:103-112.

22. Costa P, Lobo JMS. Modeling and comparison of dissolution profiles. Eur J Pharm Biopharm. 2001;13:123-133.

23. Draelos ZD, Carter E, Maloney JM, et al; United States/Canada Dapsone Gel Study Group. Two randomized studies demonstrate the efficacy and safety of dapsone gel, $5 \%$ for the treatment of acne vulgaris. $J \mathrm{Am}$ Acad Dermatol. 2007;56:439. e1-e10.

24. Jouyban A, Fakhree MAA, Shayanfar A. Review of pharmaceutical applications of N-methyl-2-pyrrolidone. J Pharm Pharm Sci. 2010;13:524-535. 
25. Zheng WW, Zhao L, Wei YM, Ye Y, Xiao SH. Preparation and the in vitro evaluation of nanoemulsion system for the transdermal delivery of granisetron hydrochloride. Chem Pharm Bull. 2010;58: 1015-1019.

26. Lee PJ, Langer R, Shastri VP. Novel nanoemulsion enhancer formulation for simultaneous transdermal delivery of hydrophilic and hydrophobic drugs. Pharm Res. 2003;20:264-269.

27. Fujii M, Koizumi A, Kinoshita Y, et al, Effect of n-methyl-2-pyrrolidone on the skin permeation of estradiol and levonorgestrel from adhesive strips prepared using Eudragit EPO. J Drug Deliv Sci Technol. 2006;16:121-125

28. Babu RJ, Pandit JK. Effect of penetration enhancers on the transdermal delivery of bupranolol through rat skin. Drug Deliv. 2005; 12:165-169.

29. Yerramsetty KM, Neely BJ, Madihally SV, Gasem KA. A skin permeability model of insulin in the presence of chemical penetration enhancer. Int J Pharm. 2010;388:13-23.

30. Kreilgaard M. Influence of nanoemulsions on cutaneous drug delivery. Adv Drug Deliv Rev. 2002;54:77-98.

31. Kogan A, Garti N. Nanoemulsions as transdermal drug delivery vehicles. Adv Colloid Interface Sci. 2006;123-126:369-385.

32. Zhu W, Yu A, Wang W, Dong R, Wu J, Zhai G. Formulation design of microemulsion for dermal delivery of penciclovir. Int J Pharm. 2008;360:184-190.

33. Tenjarla S. Nanoemulsions: an overview and pharmaceutical applications. Crit Rev Ther Drug Carrier Syst. 1999;16:461-521.
34. Formariz TP, Urban MCC, da Silva Júnior AA, Gremião MPD, Oliveira AG. Nanoemulsions and liquid crystalline phases as drug delivery systems. Braz J Pharm Sci. 2005;41:301-313. Portuguese.

35. Dziubla TD, Torjman MC, Joseph JI, Murphy-Tatum M, Lowman AM. Evaluation of porous networks of poly(2-hydroxyethyl methacrylate) as interfacial drug delivery devices. Biomaterials. 2001;22:2893-2899.

36. Scior T, Raddatz G, Figueroa R, Roth HJ, Bisswanger HA. Molecular modeling study on dapsone and sulfonamides comparing structures and properties with respect to anti-leprosy activity. J Mol Model. 1997;3:332-337.

37. Queiroz RHC, Dreossi SA, Carvalho D. A rapid, specific, and sensitive method for the determination of acetylation phenotype using dapsone. J Anal Toxicol. 1997;21:203-207.

38. British Pharmacopoeia. The official collection of standards for United Kingdom medicinal products and pharmaceutical substances. London, UK: The Stationery Office; 2005.

39. Patel MR, Patel RB, Parikh JR, Solanki AB, Patel BG. Effect of formulation components on the in vitro permeation of microemulsion drug delivery system of fluconazole. AAPS Pharm Sci Tech. 2009;10:917-923.

40. Trommer H, Neubert RHH. Overcoming the stratum corneum: the modulation of skin penetration. A review. Skin Pharmacol Physiol. 2006;19:106-121.

41. Zhu YI, Stiller MJ. Dapsone and sulfones in dermatology: overview and update. J Am Acad Dermatol. 2001;45:420-434.
International Journal of Nanomedicine

\section{Publish your work in this journal}

The International Journal of Nanomedicine is an international, peerreviewed journal focusing on the application of nanotechnology in diagnostics, therapeutics, and drug delivery systems throughout the biomedical field. This journal is indexed on PubMed Central, MedLine, CAS, SciSearch $\AA$, Current Contents ${ }^{\circledR} /$ Clinical Medicine,

\section{Dovepress}

Journal Citation Reports/Science Edition, EMBase, Scopus and the Elsevier Bibliographic databases. The manuscript management system is completely online and includes a very quick and fair peer-review system, which is all easy to use. Visit http://www.dovepress.com/ testimonials.php to read real quotes from published authors. 